\title{
Bio-guided Isolation, Purification and Chemical Characterization of Epigallocatechin; Epicatechin, Stigmasterol, Phytosterol from of Ethyl Acetate Stem Bark Fraction of Spondias mombin (Linn.)
}

\section{Oludare Temitope Osuntokun ${ }^{1 *}$, T.O Idowu ${ }^{2}$ and Gamberini Maria Cristina ${ }^{3}$}

${ }^{1}$ Department of Microbiology, Faculty of Science, Adekunle Ajasin University, Akungba Akoko, P.M.B 001, Ondo State, Nigeria

${ }^{2}$ Department of Pharmaceutical Chemistry, Obafemi Awolowo University, Nigeria

${ }^{3}$ Department of Life Sciences, University of Modena and Reggio Emilia, via G. Campi 103, 41125 Modena, Italy

\begin{abstract}
Spondias mombin (Linn.) is a widely cultivated edible plant used in folkloric medicine for the treatment of severe infection and health disorders. This research work was carried out to isolation, purification and chemical characterization the bioactive constituents of the ethyl acetate stem bark fraction of Spondias mombin (Linn.), a medicinally important plant of the Anacardiaceae family. This study revealed the presence of flavonoid and steroids, which have been found to be important hormone regulators which possess antimicrobial, anti-inflammatory, antioxidant properties. The chemical investigation resulted in the isolation of $\left(\mathrm{C}_{15} \mathrm{H}_{14} \mathrm{O}_{6}\right)$ 5, 7, 3', 4'-pentahydroxy flavanol (Epicatechin), $\left(\mathrm{C}_{15} \mathrm{H}_{14} \mathrm{O}_{7}\right.$.) Epigallocatechin $\left(\mathrm{C}_{29} \mathrm{H}_{48} \mathrm{O}\right.$.), Stigmasterol phytosterol. It is here reported isolated from Spondias mombin for the first time, this makes the Spondias mombin very important medicinal plant in Nigeria and west Africa. EGC and EC arts as a strong inhibitor of HIV replication in cultured peripheral blood cells and inhibition of HIV-1 reverse transcriptase in vitro. EGC binds directly to CD4 molecules with consequent inhibition of $\mathrm{Gp} 120$ binding and inactivate viruses in-vitro by deformation of phospholipids. Stigmasterol phytosterol have been shown to lower/reduce blood cholesterol and this lowering may reduce the risk of coronary heart disease. The structure was elucidated using two dimensional NMR spectroscopy, NMR $\left({ }^{1} \mathrm{H},{ }^{13} \mathrm{C}\right)$ spectroscopy in combination with Infra-red (IR) and Mass spectrometer (MS) spectra data.
\end{abstract}

Keywords: Epigallocatechin; Epicatechin; Stigmasterol; Phytosterol; Isolation; Purification; Chemical Characterization Ethyl acetate; Spondias mombin (Linn.)

\section{Introduction}

Plants are the basis of traditional medicine in Africa and have been used for thousands of years. These plants often exhibit a wide range of pharmacological activities [1]. Due to the need for development of new drugs with better pharmacological activities, dependence on plants grew increasingly as scientists continuously exploited them for isolation of bioactive compound.

Spondias mombin is a small tree that grows up to $20 \mathrm{~m}(60 \mathrm{ft}$.) high and $1.5 \mathrm{~m}(5 \mathrm{ft})$ in girth, moderately buttressed; bark thick, corky, deeply fissured, slash pale pink, darkening rapidly, branches low, branchlets glabrous; leaves pinnate, leaflets 5-8 opposite pairs with a terminal leaflet. It belongs to the family Anacardiaceae. It flowers between January to May and fruits between July to September. The fruits have a sharp, somewhat acid taste and are edible. The matured fruit has a leathery skin and a thin layer of pulp. The fruit pulp is either eaten fresh, or made into juice, concentrate, jellies, and sherbets. The fruit-juice is used as a febrifuge and diuretic. The roots are also used as febrifuge in Ivory Coast. The stem bark is used as a purgative and in local applications for leprosy. The stem bark decoction is also used in the treatment of severe cough. It serves as an emetic, a remedy for diarrhea, dysentery, haemorrhoids and a treatment for gonorrhoea and leucorrhea [2].

A report showed that the bark contains a certain amount of tannin and this explains the reason why the dry pulverized bark is applied as a dressing to a wound [2]. In Belize, a decoction of the young leaves is a remedy for diarrhea and dysentery. The juice of crushed leaves and the powder of dried leaves are used as poultices on wounds and inflammations. The gum is employed as an expectorant and to expel tapeworms [3].
A decoction of the mashed leaves is used by the Ibos (Nigeria) for washing a swollen face. A leaf infusion is a common cough remedy or used as a laxative for fever with constipation. A leaf decoction is used in treatment of gonorrhea. The leaves are used in Ivory Coast for fresh wounds to prevent inflammation. A decoction of pounded leaves of S. mombin is used as an eye lotion and the juice pressed from young, warm leaves is given to children for stomach troubles. The extract has shown anti-inflammatory activity in Wistar rats [4]. A tea made from the flowers and leaves is taken to relieve stomach ache, biliousness, and urethritis, cystitis and eye and throat inflammations. A decoction of the root is used as purgative [4].

\section{Material and Methods}

\section{Collection of plant materials}

The stem bark of Spondias mombin (Linn.) was collected from a local farm at Owo (710'59.998N and 534'59.988E), Ondo State, Nigeria at WAT UTC +1 time zone on $26^{\text {th }}$ and $27^{\text {th }}$ of February, 2016. Fresh and healthy plants were also collected during its fruiting season, between April and July 2017, from the same geographical locations.

${ }^{*}$ Corresponding author: Osuntokun OT, Department of Microbiology, Faculty of Science, Adekunle Ajasin University, Akungba Akoko, P.M.B 001, Ondo State Nigeria, Tel: 08063813635; E-mail: osuntokun4m@gmail.com

Received: December 20, 2017; Accepted: January 31, 2018; Published February 15, 2018

Citation: Osuntokun OT, Idowu TO, Cristina GM (2018) Bio-guided Isolation, Purification and Chemical Characterization of Epigallocatechin; Epicatechin, Stigmasterol, Phytosterol from of Ethyl Acetate Stem Bark Fraction of Spondias mombin (Linn.). Biochem Pharmacol (Los Angel) 7: 240. doi: 10.4172/2167-0501.1000240

Copyright: (๑) 2018 Osuntokun OT, et al. This is an open-access article distributed under the terms of the Creative Commons Attribution License, which permits unrestricted use, distribution, and reproduction in any medium, provided the original author and source are credited. 
Citation: Osuntokun OT, Idowu TO, Cristina GM (2018) Bio-guided Isolation, Purification and Chemical Characterization of Epigallocatechin; Epicatechin, Stigmasterol, Phytosterol from of Ethyl Acetate Stem Bark Fraction of Spondias mombin (Linn.). Biochem Pharmacol (Los Angel) 7: 240. doi: 10.4172/2167-0501.1000240

Page 2 of 9

\section{Authentication of Spondias mombin (Linn.)}

The plants were authenticated at the Department of Plant Science and Biotechnology, Adekunle Ajasin University, Akungba- Akoko, Ondo state, Nigeria.

\section{Preparation and extraction of Spondias mombin (Linn.) Plant}

The stem-bark of Spondias mombin plant were harvested and airdried. The dried stem bark were milled into powdered form using manual grinder. Powdered plant material $(1 \mathrm{~kg})$ each of the different plant parts was extracted with $3 \mathrm{~L}$ of $70 \%(\mathrm{v} / \mathrm{v})$ ethanol, ethyl acetate and distilled water for $72 \mathrm{~h}$ at room temperature. The extraction process was repeated four times until the extract became clear. The filtrates were combined and concentrated under reduced pressure rotatory evaporator at $35^{\circ} \mathrm{C}$ to give, SMSBEA for the stem-bark part. The dry extracts were kept in tightly stoppered bottles in a refrigerator at $-4^{\circ} \mathrm{C}$ for further analysis

\section{Fractionation of SMSBEAEA on column Chromatography}

The ethyl acetate fraction SMSBEAEA $(11.0 \mathrm{~g})$ was adsorbed unto silica and allowed to dry before packing on to column. The column was wetted with $n$-hexane and gradient elution effected with the following solvent/ solvent mixtures (Table 1).

Fractions collected were analysed by TLC (Thin layer chromatography) in Hex - EtOAc - AcOH (1: 9: 0.5). The resulting spots on TLC plates 1-3 were visualized under UV light $(254 \mathrm{~nm})$ and detected by the use of vanillin/sulfuric acid and the antioxidant compound(s) were detected using DPPH spray reagents. Fractions having the same TLC patterns were bulked, concentrated in vacuo to dryness and weighed; resulting in three fractions coded SMSBEAEA1, SMSBEAEA2 and SMSBEAEA3 (Table 2).

\section{Fractionation of SMSBEAEA1 on Sephadex LH-20}

Fraction SMSBEAEA1 (1.6 g) was dissolved in a small amount of $\mathrm{CHCl}_{3}-\mathrm{MeOH}$ (70:30) solvent mixture and loaded on a Sephadex LH20 column previously equilibrated with the same solvent mixture and

\begin{tabular}{|c|c|c|c|}
\hline Hexane & $100 \%$ & $200 \mathrm{ml}$ & $1-7$ \\
\hline Hexane - ethyl acetate & $(80: 20)$ & $100 \mathrm{ml}$ & $8-12$ \\
\hline Hexane - ethyl acetate & $(70: 30)$ & $200 \mathrm{ml}$ & $13-22$ \\
\hline Hexane - ethyl acetate & $(60: 40)$ & $100 \mathrm{ml}$ & $23-27$ \\
\hline Hexane - ethyl acetate & $(50: 50)$ & $200 \mathrm{ml}$ & $28-37$ \\
\hline Hexane - ethyl acetate & $(30: 70)$ & $100 \mathrm{ml}$ & $38-42$ \\
\hline Hexane - ethyl acetate & $(20: 80)$ & $200 \mathrm{ml}$ & $43-52$ \\
\hline Hexane - ethyl acetate & $(10: 90)$ & $100 \mathrm{ml}$ & $53-57$ \\
\hline Ethyl acetate & $100 \%$ & $200 \mathrm{ml}$ & $58-67$ \\
\hline Ethyl acetate - Methanol & $(95: 5)$ & $200 \mathrm{ml}$ & $68-77$ \\
\hline Ethyl acetate - Methanol & $(90: 10)$ & $200 \mathrm{ml}$ & $78-87$ \\
\hline Ethyl acetate - Methanol & $80-20$ & $200 \mathrm{ml}$ & $88-97$ \\
\hline Ethyl acetate - Methanol & $75-25$ & $100 \mathrm{ml}$ & $98-102$ \\
\hline Ethyl acetate - Methanol & $70-30$ & $200 \mathrm{ml}$ & $103-112$ \\
\hline Ethyl acetate - Methanol & $65-35$ & $200 \mathrm{ml}$ & $113-122$ \\
\hline
\end{tabular}

Table 1. Fractionation of SMSBEAEA on Column Chromatography with Hexane/ ethyl acetate/ Methanol solvent.

\begin{tabular}{|c|c|c|}
\hline Fractions & Codes & Weight \\
\hline $1-67$ & SMSBEAEA1 & $1.6 \mathrm{~g}$ \\
\hline $68-77$ & SMSBEAEA2 & $0.1 \mathrm{~g}$ \\
\hline $78-122$ & SMSBEAEA3 & $0.2 \mathrm{~g}$ \\
\hline
\end{tabular}

Table 2: Three fractions coded SMSBEAEA1, SMSBEAEA2 and SMSBEAEA3 final Weight after Column Chromatography. elution was isocratically effected. Fractions (about $20 \mathrm{ml}$ each) collected were analysed by TLC in $\mathrm{CH}_{2} \mathrm{Cl}_{2}-\mathrm{MeOH}$ (10: 1.0) and fractions having the similar TLC patterns were bulked together, concentrated in vacuo to dryness and weighed; resulting in eight fractions coded SMSBEAEA1a, SMSBEAEA1b, SMSBEAEA1c (compound 1), SMSBEAEA1d, SMSBEAEA1e (compound 2), SMSBEAEA1f, SMSBEAEA1g and SMSBEAEA1h (Table 3).

\section{Fractionation of SMSBEAEA1a on column Chromatography}

Fraction SMSBEAEA1a (600 g) was adsorbed unto silica and allowed to dry before packing on to column. The column was wetted with $n$-hexane and gradient elution effected with the following solvent/ solvent mixtures (Table 4):

Fractions (about $20 \mathrm{ml}$ each) collected were analysed by TLC in hexane - $\mathrm{CH}_{2} \mathrm{Cl}_{2}(8: 4)$ and hexane $-\mathrm{CH}_{2} \mathrm{Cl}_{2}(2: 8)$ and fractions having the similar TLC patterns were bulked together, concentrated in vacuo to dryness and weighed resulting in six fractions coded SMSBEAEA1a1, SMSBEAEA1a2, SMSBEAEA1a3, SMSBEAEA1a4 (3), SMSBEAEA1a5 and SMSBEAEA1a6 (Table 5 and Figure 1).

\section{Result}

The physical and spectroscopic data on the isolated compounds are as recorded below.

\begin{tabular}{|c|c|c|}
\hline Fractions & Codes & Weight \\
\hline $1-7$ & SMSBEAEA1a & $623 \mathrm{mg}$ \\
\hline $8-9$ & SMSBEAEA1b & $68 \mathrm{mg}$ \\
\hline $10-14$ & SMSBEAEA1c (1) & $230 \mathrm{mg}$ \\
\hline $14-15$ & SMSBEAEA1d & $97 \mathrm{mg}$ \\
\hline 16 & SMSBEAEA1e (2) & $15 \mathrm{mg}$ \\
\hline $17-21$ & SMSBEAEA1f & $155 \mathrm{mg}$ \\
\hline $22-26$ & SMSBEAEA1g & $180 \mathrm{mg}$ \\
\hline $27-31$ & SMSBEAEA1h & $62 \mathrm{mg}$ \\
\hline
\end{tabular}

Table 3. Eight fractions coded SMSBEAEA1a, SMSBEAEA1b, SMSBEAEA1c (compound 1), SMSBEAEA1d, SMSBEAEA1e (compound 2), SMSBEAEA1f, SMSBEAEA1g and SMSBEAEA1h final Weight on Sephadex LH-20.

\begin{tabular}{|c|c|c|c|}
\hline Hexane & $100 \%$ & $100 \mathrm{ml}$ & $1-3$ \\
\hline Hexane - dichloromethane & $(95: 5)$ & $100 \mathrm{ml}$ & $4-8$ \\
\hline Hexane - dichloromethane & $(90: 10)$ & $500 \mathrm{ml}$ & $9-31$ \\
\hline Hexane - dichloromethane & $(85: 15)$ & $500 \mathrm{ml}$ & $32-52$ \\
\hline Hexane - dichloromethane & $(80: 20)$ & $300 \mathrm{ml}$ & $53-66$ \\
\hline Hexane - dichloromethane & $(70: 30)$ & $300 \mathrm{ml}$ & $67-79$ \\
\hline Hexane - dichloromethane & $(50: 50)$ & $200 \mathrm{ml}$ & $80-89$ \\
\hline Hexane - dichloromethane & $(30: 70)$ & $200 \mathrm{ml}$ & $90-98$ \\
\hline Hexane - dichloromethane & $(10: 90)$ & $200 \mathrm{ml}$ & $99-107$ \\
\hline Dichloromethane & $100 \%$ & $200 \mathrm{ml}$ & $108-117$ \\
\hline
\end{tabular}

Table 4: Fractionation of SMSBEAEA on Column Chromatography with Hexane/ dichloromethane solvent

\begin{tabular}{|c|c|c|}
\hline Fractions & Codes & Weight. \\
\hline $1-52$ & SMSBEAEA1a1 & $196 \mathrm{mg}$ \\
\hline $53-75$ & SMSBEAEA1a2 & $121 \mathrm{mg}$ \\
\hline $76-87$ & SMSBEAEA1a3 & $95 \mathrm{mg}$ \\
\hline $88-91$ & SMSBEAEA1a4 (3) & $23 \mathrm{mg}$ \\
\hline $98-105$ & SMSBEAEA1a5 & $60 \mathrm{mg}$ \\
\hline $106-117$ & SMSBEAEA1a6 & $35 \mathrm{mg}$ \\
\hline
\end{tabular}

Table 5: Six fractions coded SMSBEAEA1a1, SMSBEAEA1a2, SMSBEAEA1a3 SMSBEAEA1a4 (3), SMSBEAEA1a5 and SMSBEAEA1a6 final Weight on Sephadex LH-20 
Citation: Osuntokun OT, Idowu TO, Cristina GM (2018) Bio-guided Isolation, Purification and Chemical Characterization of Epigallocatechin; Epicatechin, Stigmasterol, Phytosterol from of Ethyl Acetate Stem Bark Fraction of Spondias mombin (Linn.). Biochem Pharmacol (Los Angel) 7: 240. doi: 10.4172/2167-0501.1000240

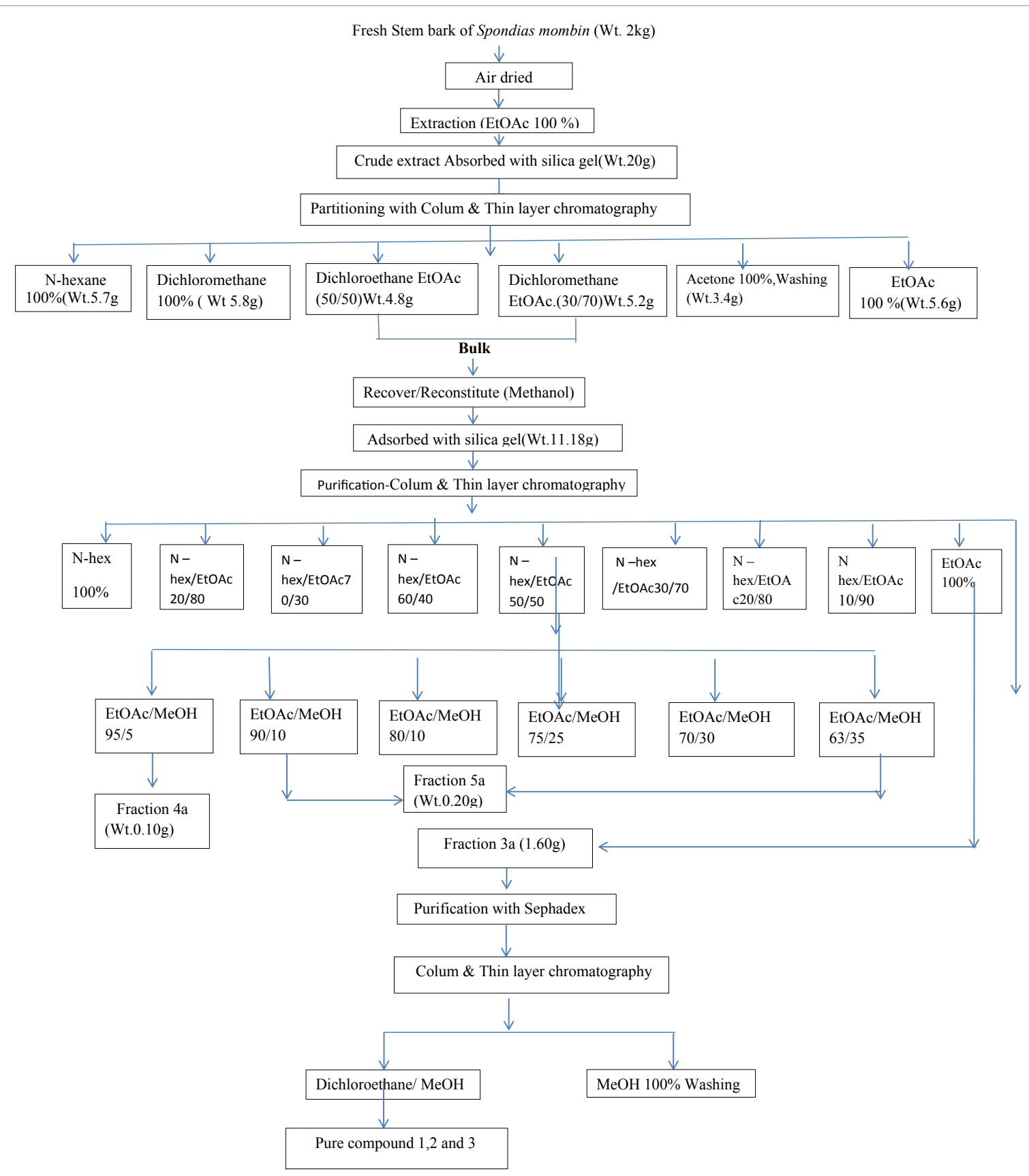

Figure 1: Isolation and purification of active compounds from the bioactive fractions (Pure compound) Key: 1- Epigallocatechin; 2- Epicatechin and 3-Stigmastero Phytosterol.

\section{Characterization of compound 1}

Physical characteristics: Pale yellow powder, m.p. $240-242^{\circ} \mathrm{C}$ (decomp); $[\alpha]_{\mathrm{D}}{ }^{20}-60\left(\mathrm{CD}_{3} \mathrm{OD}, \mathrm{c}=1.00\right) ; \mathrm{ESI}-\mathrm{MS} \mathrm{m} / \mathrm{z} 291[\mathrm{M}+\mathrm{H}]^{+}, 139$ [M+H-152] $]^{+}$IR $V \max \mathrm{KBr} \mathrm{cm}^{-1}: 3456.2,1620.10,1521.70,1450.40,1265.2$ 1143.7; UV $\lambda \max \mathrm{MeOH} n m$ 213.00, 280; ${ }^{1} \mathrm{H}$ - and ${ }^{13} \mathrm{C}-\mathrm{NMR}$ (CD $\mathrm{OD}$ ).

Spectroscopic data 1: ${ }^{1} \mathrm{Hnmr}\left(300 \mathrm{MHz}, \mathrm{CD}_{3} \mathrm{OD}\right) \delta$ ppm: 4.89 $(1 \mathrm{H}, \mathrm{bs}, \mathrm{H}-2), 4.01(1 \mathrm{H}, \mathrm{bs}, \mathrm{H}-3), 2.88(1 \mathrm{H}$ dd, J=4.1, $12.15 \mathrm{~Hz}, \mathrm{H}-4 \mathrm{a})$, 2.84, $1 \mathrm{H}_{\mathrm{b},} d d,(4.5,12.09 \mathrm{~Hz}, \mathrm{H}-4 \mathrm{~b}), 5.89(1 \mathrm{H}, d, J=2.2 \mathrm{~Hz}, \mathrm{H}-6), 5.97$ $(1 \mathrm{H}, d, J=2.2 \mathrm{~Hz}, \mathrm{H}-8), 7.09\left(1 \mathrm{H}, \mathrm{d}, J=2.2 \mathrm{~Hz}, \mathrm{H}-2^{\prime}\right), 6.74(1 \mathrm{H}, \mathrm{d}, J=$ $\left.6.00 \mathrm{~Hz}, \mathrm{H}-5^{\prime}\right), 6.79\left(1 \mathrm{H}, \mathrm{dd}, J=6.2,1.9 \mathrm{~Hz}, \mathrm{H}-6^{\prime}\right)$.
Spectroscopic data 2: ${ }^{13} \mathrm{Cnmr}\left(75 \mathrm{MHz}, \mathrm{CD}_{3} \mathrm{OD}\right) \delta \mathrm{ppm}: 82.8(\mathrm{C}-$ 2), 68.8 (C-3), 28.5 (C-4), 157.5 (C-5), 95.6 (C-6), 157.7 (C-7), 96.5 (C8), 156.8 (C-9), $101.0(\mathrm{C}-10), 132.2\left(\mathrm{C}-1^{\prime}\right), 115.4\left(\mathrm{C}-2^{\prime}\right), 146.2\left(\mathrm{C}-3^{\prime}\right)$, $146.4\left(\mathrm{C}-4^{\prime}\right), 116.3\left(\mathrm{C}-5^{\prime}\right), 120.2\left(\mathrm{C}-6^{\prime}\right)$.

The ${ }^{1} \mathrm{Hnmr},{ }^{13} \mathrm{Cnmr}$, DEPT, HMQC, HMBC, MS, IR and UV spectra are provided in Figure 1.

\section{Characterization of compound 2}

Physical characteristics: Brown amorphous powder, $\left[_{\mathrm{D}}{ }^{20} 110^{0}\right.$ $\left(\mathrm{Me}_{2} \mathrm{CO}, \mathrm{c}=1.00\right) ;$ EIMS m/z ESI-MS m/z $345[\mathrm{M}+\mathrm{K}]^{+}, 139[\mathrm{M}+\mathrm{H}-$ 
Citation: Osuntokun OT, Idowu TO, Cristina GM (2018) Bio-guided Isolation, Purification and Chemical Characterization of Epigallocatechin; Epicatechin, Stigmasterol, Phytosterol from of Ethyl Acetate Stem Bark Fraction of Spondias mombin (Linn.). Biochem Pharmacol (Los Angel) 7: 240. doi: 10.4172/2167-0501.1000240

$168]^{+} ;$IR $V_{\max } \mathrm{KBr} \mathrm{cm}^{-1}: 3345,2923.45 .9,1614.3,1519.8,1463.9,1353.9$, 1282.6, $1195.8 ;{ }^{1} \mathrm{H}-$ and 13C-NMR (CD3OD).

Spectroscopic data 1: ${ }^{1} \mathrm{Hnmr}\left(300 \mathrm{MHz}, \mathrm{CD}_{3} \mathrm{OD}\right) \delta$ ppm: 4.89 (1H, bs, H-2), $4.01(1 \mathrm{H}, \mathrm{bs}, \mathrm{H}-3), 2.88\left(1 \mathrm{H}_{\mathrm{a}} d d, J=4.1,12.15 \mathrm{~Hz}, \mathrm{H}-4 \mathrm{a}\right)$, $2.84,1 \mathrm{H}_{\mathrm{b},} d d,(4.5,12.09 \mathrm{~Hz}, \mathrm{H}-4 \mathrm{~b}), 5.89(1 \mathrm{H}, d, J=2.2 \mathrm{~Hz}, \mathrm{H}-6), 5.97$ $(1 \mathrm{H}, d, J=2.2 \mathrm{~Hz}, \mathrm{H}-8), 6.9\left(2 \mathrm{H}, \mathrm{s}, \mathrm{H}-2^{\prime} / \mathrm{H}-6^{\prime}\right)$.

Spectroscopic data 2: ${ }^{13} \mathrm{Cnmr}\left(75 \mathrm{MHz}, \mathrm{CD}_{3} \mathrm{OD}\right) \delta \mathrm{ppm}: 82.8(\mathrm{C}-$ 2), 68.8 (C-3), 28.5 (C-4), 157.5 (C-5), 95.6 (C-6), 157.7 (C-7), 96.5 (C8), 156.8 (C-9), 101.0 (C-10), 132.2 (C-1'), 110.5 (C-2'), 146.2 (C-3'), $132.6\left(\mathrm{C}-4^{\prime}\right), 146.4\left(\mathrm{C}-5^{\prime}\right), 110.5\left(\mathrm{C}-6^{\prime}\right)$.

The ${ }^{1} \mathrm{Hnmr},{ }^{13} \mathrm{Cnmr}$, COSY, DEPT, HMQC, HMBC, MS, IR and UV spectra are provided as Figure 2.

\section{Characterization of compound 3}

Physical characteristics: White crystal, ESI-MS $m / z:[M+H] 413$, ${ }^{1} \mathrm{H}$ - and ${ }^{13} \mathrm{C}-\mathrm{NMR}\left(\mathrm{CDCl}_{3}\right)$

Spectroscopic data 1: ${ }^{13} \mathrm{Cnmr}\left(75 \mathrm{MHz}, \mathrm{CDCl}_{3}\right) \mathrm{d}$ ppm: 30.79 (C1), 30.18 (C-2), 69.26 (C-3), 38.42 (C-4), 146.29 (C-5), 117.74 (C-6), 37.56 (C-7), 41.12 (C-8), 48.78 (C-9), 31.66 (C-10), 21.65 (C-11), 39.34 (C-12), 42.63 (C-13), 56.51 (C-14), 24.08 (C-15), 25.00 (C-16), 54.52 (C-17), 12.87 (C-18), 14.56 (C-19), 40. 80 (C-20), 21.49 (C-21), 132.46 (C-22), 129.94 (C-23), 51.35 (C-24), 30.99 (C-25), 21.29 (C-26), 19.14 (C-27), 25.23 (C-28), 11.57 (C29) (Figures 3-5).

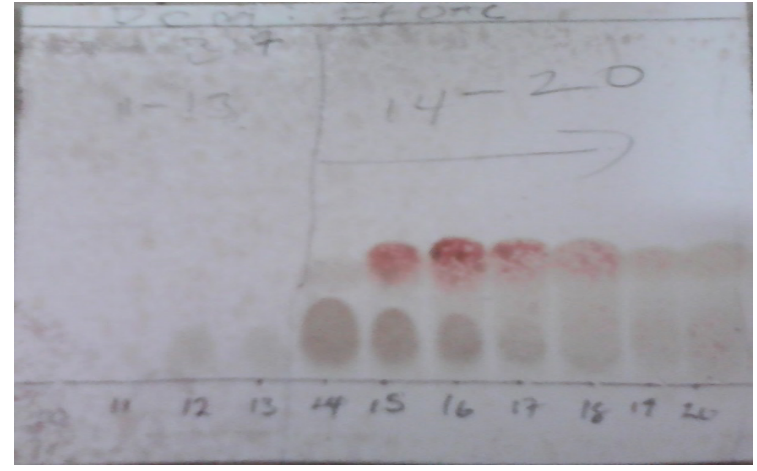

TLC Plate 1: Isolation, Identification and Characterization of Epigallocatechin and Epicatechin.

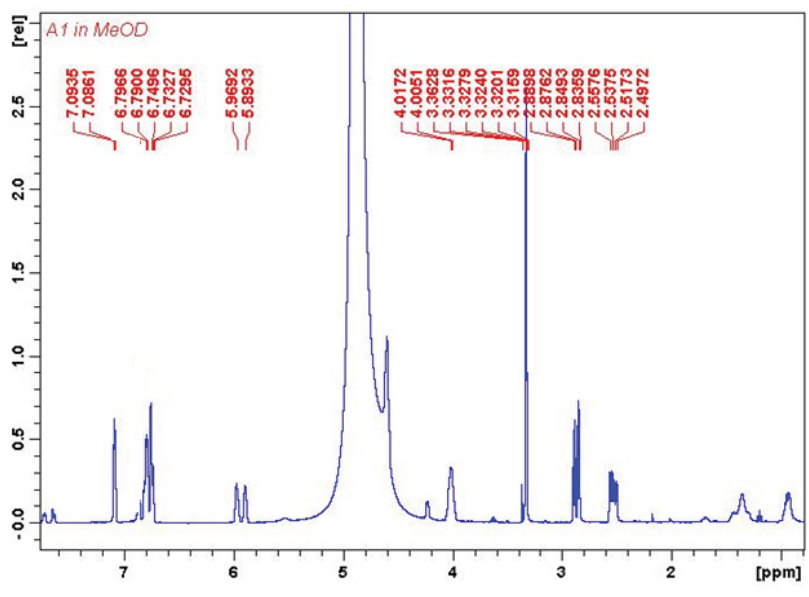

Figure 2: MeOD of Epicatechin
Spectroscopic data 1: The ${ }^{13} \mathrm{Cnmr}$, MS, spectra (fig 2). ${ }^{13} \mathrm{Cnmr}(75$ $\left.\mathrm{MHz}, \mathrm{CD}_{3} \mathrm{OD}\right) \delta$ ppm: 82.8 (C-2), 68.8 (C-3), 28.5 (C-4), 157.5 (C-5), 95.6 (C-6), 157.7 (C-7), 96.5 (C-8), 156.8 (C-9), 101.0 (C-10), 132.2 (C1'), $110.5\left(\mathrm{C}-2^{\prime}\right), 146.2\left(\mathrm{C}-3^{\prime}\right), 132.6\left(\mathrm{C}-4^{\prime}\right), 146.4\left(\mathrm{C}-5^{\prime}\right), 110.5\left(\mathrm{C}-6^{\prime}\right)$.

\section{Discussion}

This research work is based on the isolation, purification and elucidation of structure (Chemical characterization) of the isolated constituents from Spondias mombin extract. It was observed that, three different bioactive constituents were isolated, purified and structurally
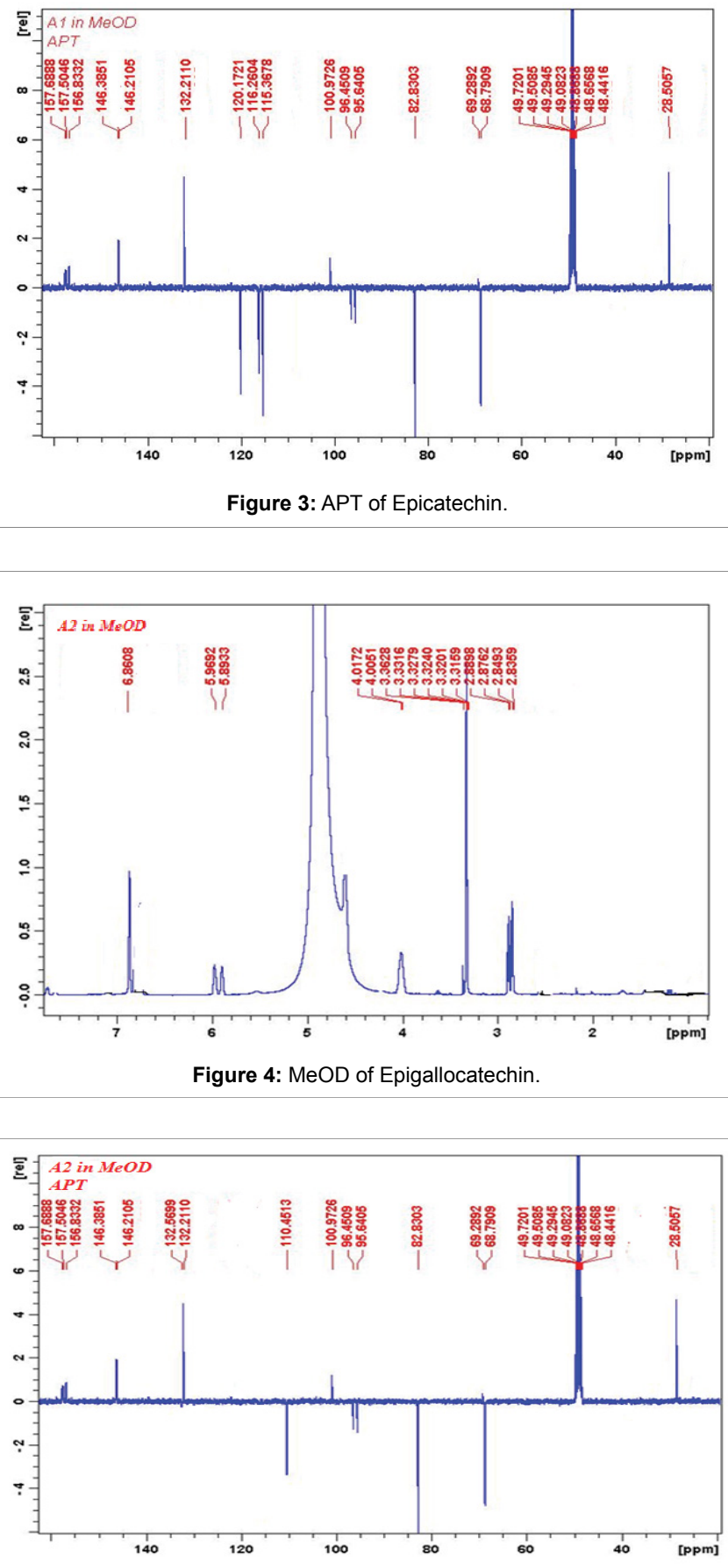

Figure 5: APT of Epigallocatechin 
Citation: Osuntokun OT, Idowu TO, Cristina GM (2018) Bio-guided Isolation, Purification and Chemical Characterization of Epigallocatechin; Epicatechin, Stigmasterol, Phytosterol from of Ethyl Acetate Stem Bark Fraction of Spondias mombin (Linn.). Biochem Pharmacol (Los Angel) 7: 240. doi: 10.4172/2167-0501.1000240

Page 5 of 9

elucidated from the stem bark extract of Spondias mombin. They are Epigallocatechin, Epicatechin and Stigmasterol Phyto sterol.

\section{Identification ofCompound 1 as 3, 5,7,3',4'-pentahydroxyflavanol (Epicatechin)}

From the ethyl acetate fraction of the stem bark, compound 1 was isolated as yellow solid. The negative ESI -MS spectrum of compound 1 showed ion peak at $m / z 291[\mathrm{M}+\mathrm{H}]^{+}$corresponding to the molecular formula $\mathrm{C}_{15} \mathrm{H}_{14} \mathrm{O}_{6}$. In the same MS spectrum, fragment ion found at $m / z$ 139 resulted from a retro-diels-Alder (RDA) fragmentation. Analyzing the ${ }^{13} \mathrm{C}$ NMR and ${ }^{1} \mathrm{H}$ NMR spectral data revealed presence of two aromatic systems one exhibiting an $\mathrm{ABD}$ proton spin system with protons resonating at $\delta_{\mathrm{H}} 7.09(1 \mathrm{H}, d, J=1.8 \mathrm{~Hz}), \delta_{\mathrm{H}} 6.74(1 \mathrm{H}, d, J=6.0$

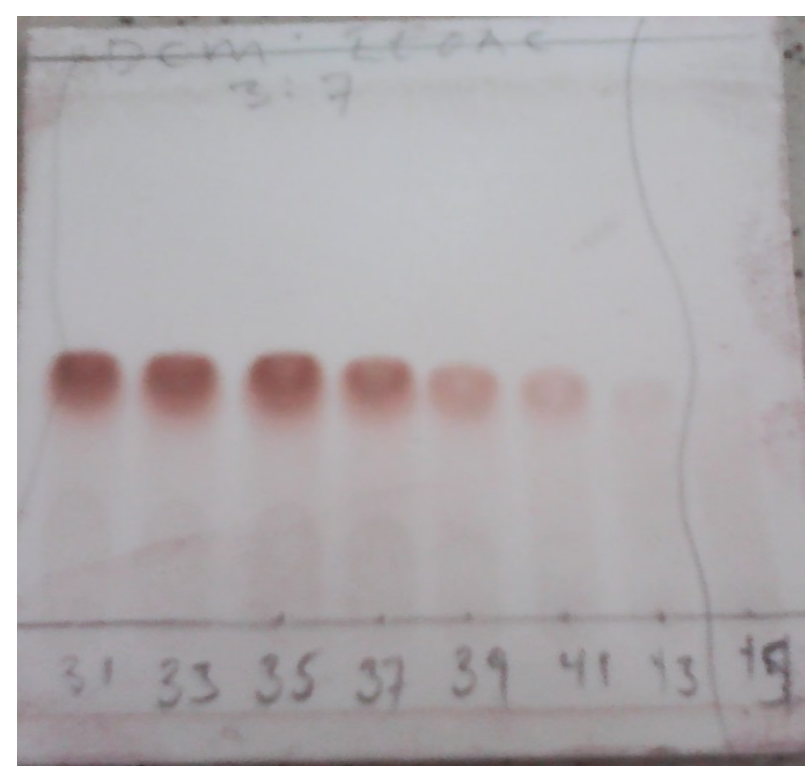

TLC Plate 2: Isolation, Identification and Characterization of Stigmastero Phytosterol.

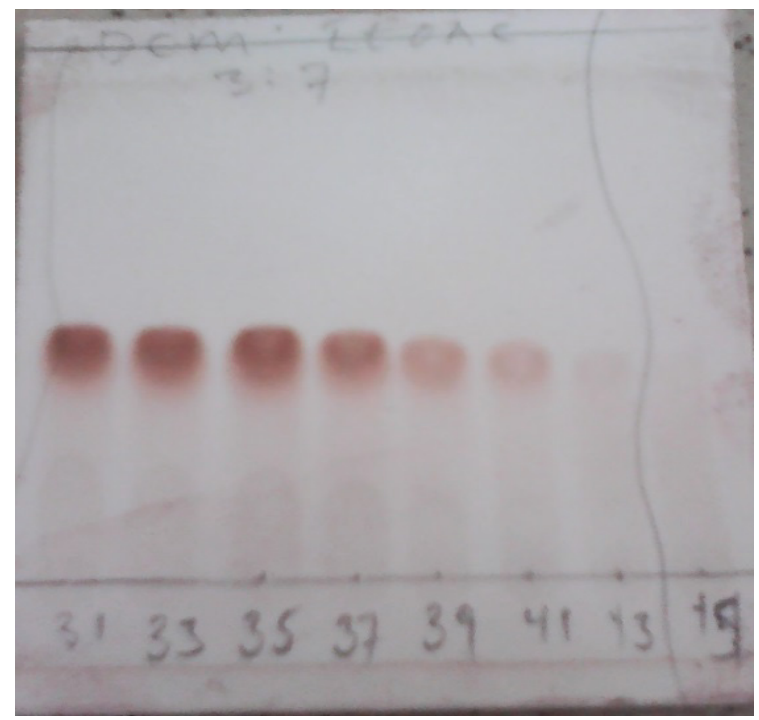

TLC Plate 3: Isolation, Identification and Characterization of Stigma sterol Phytosterol.
$\mathrm{Hz})$ and $6.79(1 \mathrm{H}, d d, J=6.2,1.9 \mathrm{~Hz})$ assignable to $\mathrm{H}-2^{\prime}, \mathrm{H}^{-} 5^{\prime}$ and $\mathrm{H}-6$ protons attached on carbons $\mathrm{C}-2^{\prime}\left({ }_{\mathrm{C}} 115.4\right), \mathrm{C}-5^{\prime}\left(\delta_{\mathrm{C}} 116.3\right)$ and C-6' $\left(\delta_{\mathrm{C}}\right.$ $120.2)$ on ring $B$ respectively. The $\mathrm{HMBC}$ spectrum showed that $\mathrm{H}-2$ proton correlated with carbons $\mathrm{C}-2\left(\delta_{\mathrm{C}} 78.9\right), \mathrm{C}-3^{\prime}\left(\delta_{\mathrm{C}} 146.2\right), \mathrm{C}-4^{\prime}\left(\delta_{\mathrm{C}}\right.$ 146.4), C-5' and C-6' whereas $\mathrm{H}-6$ proton showed correlations with carbons C-1' $\left(\delta_{\mathrm{C}} 132.2\right), \mathrm{C}-2, \mathrm{C}-3^{\prime}, \mathrm{C}-4^{\prime}, \mathrm{C}-5^{\prime}$ and C-6' (Figures 6-13).

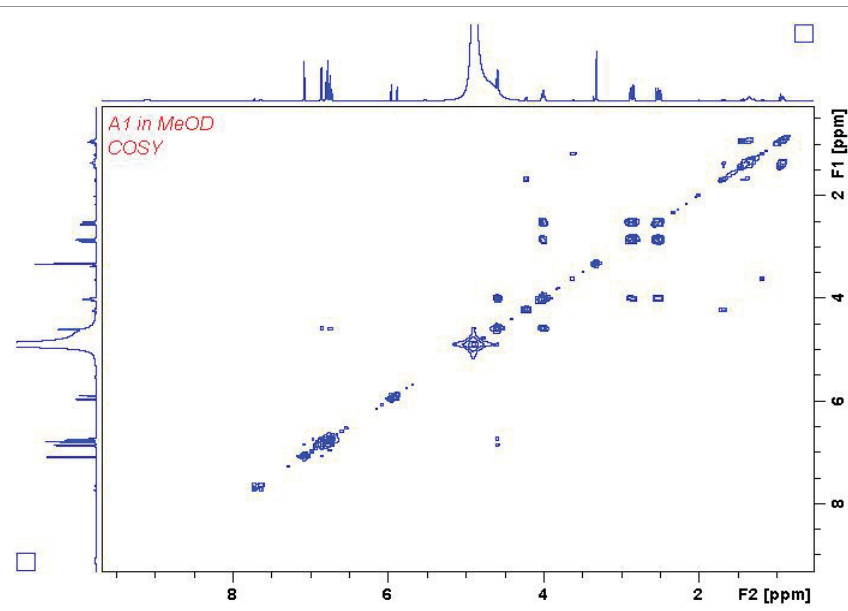

Figure 6: COSY analysis of compounds isolated from Stem bark extract of Spondias mombin.

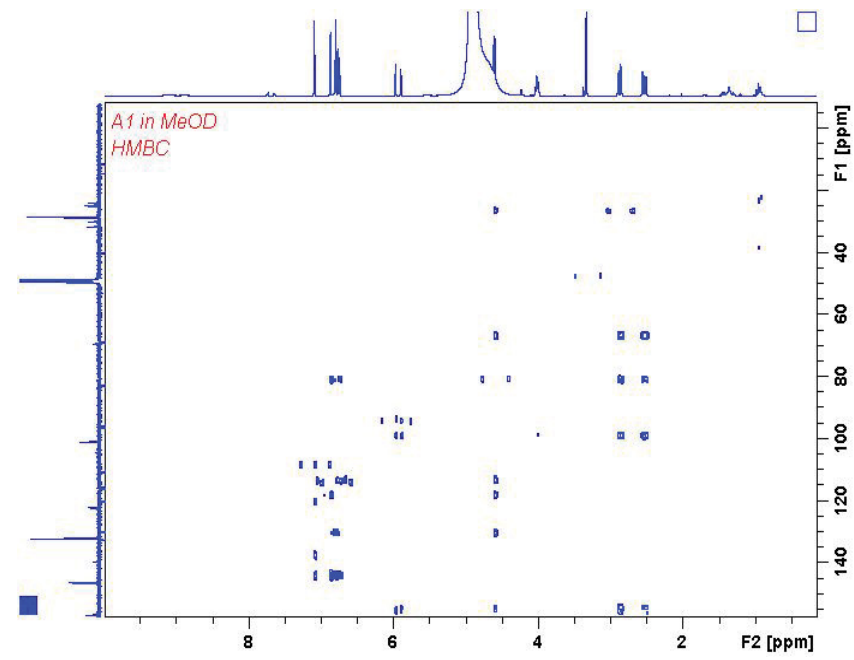

Figure 7: HMBC analysis of compounds isolated from Stem bark extract of Spondias mombin.

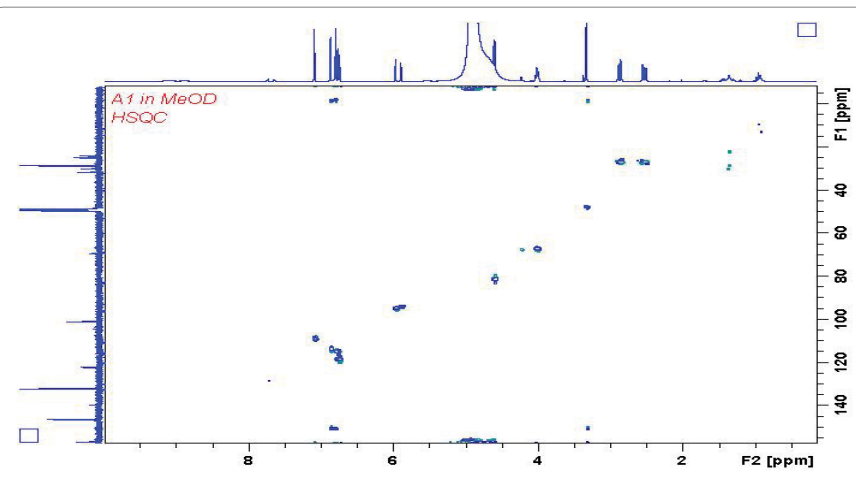

Figure 8: HSQC analysis of compounds isolated from Stem bark extract of Spondias mombin. 
Citation: Osuntokun OT, Idowu TO, Cristina GM (2018) Bio-guided Isolation, Purification and Chemical Characterization of Epigallocatechin; Epicatechin, Stigmasterol, Phytosterol from of Ethyl Acetate Stem Bark Fraction of Spondias mombin (Linn.). Biochem Pharmacol (Los Angel) 7: 240. doi: 10.4172/2167-0501.1000240

Page 6 of 9

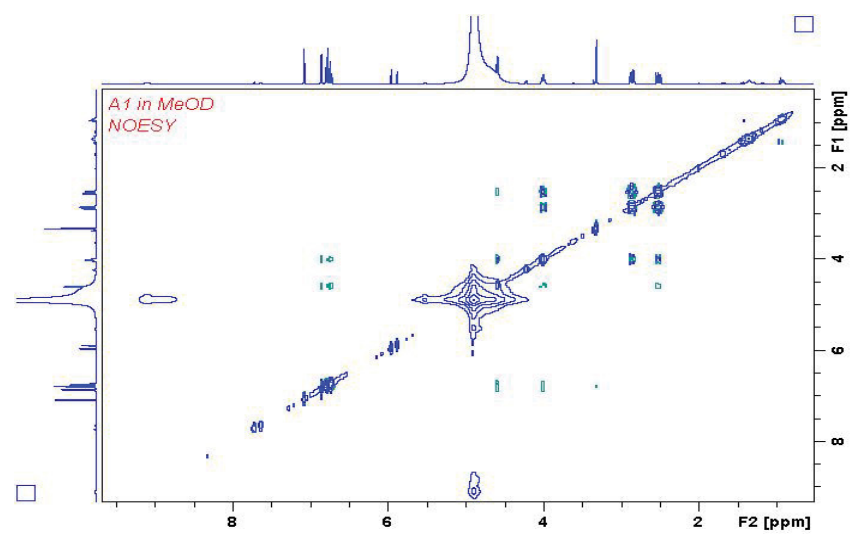

Figure 9: Noesy analysis of compounds isolated from Stem bark extract of Spondias mombin.

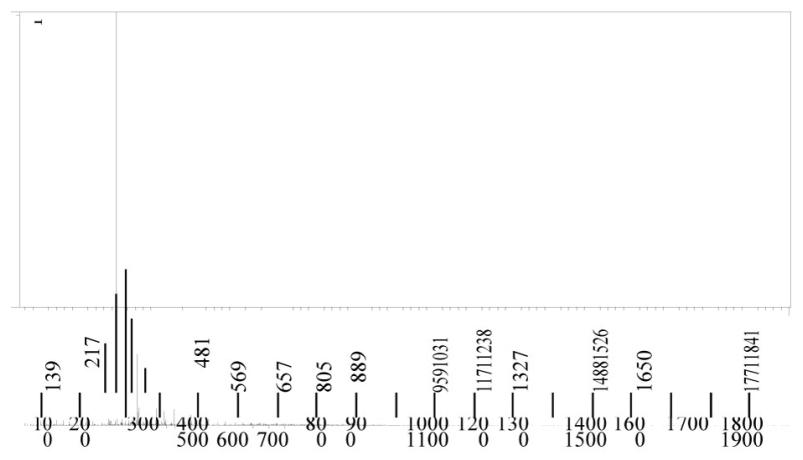

Figure 10: MS analysis of compound A1 isolated from Stem bark extract of Spondias mombin (SpectrumR.Time:1.067(Scan\#:65) MassPeaks:2005).
Furthermore the HMBC spectrum showed correlations of $\mathrm{H}^{-5}$ proton with carbons $\mathrm{C}-1^{\prime}, \mathrm{C}-3^{\prime}$ and $\mathrm{C}-4$ '. Thus the $\mathrm{ABD}$ proton spin system was placed on ring $\mathrm{B}$. Ring $\mathrm{C}$ on the other hand displayed a saturated system with two germinal protons resonating at $\delta_{\mathrm{H}} 2.88$ $\left(1 \mathrm{H}_{\mathrm{a}}, d d, J=4.1,12.15 \mathrm{~Hz}\right)$ and $2.84\left(1 \mathrm{H}_{\mathrm{b}}, d d, J=4.5,12.09 \mathrm{~Hz}\right)$ and they were placed at C-4 $\left(\delta_{C} 28.5\right)$ based on DEPT, HMQC and HMBC experiments. The negative ESI -MS fragment ion at $\mathrm{m} / z 139$ resulting from a retro Diels - Alder fragmentation (Scheme 1) confirmed that carbon C-4 consists of two protons consistent with the flavan skeleton. The absence of carbonyl absorption peaks in the ${ }^{13} \mathrm{C}$ NMR spectrum (Figures 6-9) on the region $170-210 \mathrm{ppm}$ (Agrawal,1989) and IR around $1700-1750 \mathrm{~cm}^{-1}$ confirmed the proposed flavanol skeleton. The second aromatic ring exhibited a proton spin system comprised of meta-coupled protons with resonances observed at $\delta_{\mathrm{H}} 5.89(1 \mathrm{H}, d$, $J=2.2 \mathrm{~Hz})$ and $5.97(1 \mathrm{H}, d, J=2.2 \mathrm{~Hz})$ which were assigned to $\mathrm{H}-6$ and H-8 protons placed on carbons C-6 $\left({ }_{C} 95.6\right)$ and C-8 $\left(\delta_{C} 96.5\right)$ respectively based on HMBC, DEPT and HMQC experiments, this proton spin system was thus placed on ring $\mathrm{A}$.

From the above information compound $\mathbf{1}$ was identified as 3,5 , 7, 3', 4'-pentahydroxyflavanol, commonly known as (-) - epicatechin, a widely distributed compound in the plant kingdom and has been reported to be responsible for anti-inflammatory and antioxidant properties in green teas [5] and other plant extracts. However this is the first time we report its occurrence from Spondias mombin (Scheme 1).

${ }^{1} \mathrm{H}$ and ${ }^{13} \mathrm{C}$ NMR Data of Compound 1 were listed as follows Position $2\left(\delta_{\mathrm{H}}-4.89,1 \mathrm{H}\right.$, br s $)\left(\delta_{\mathrm{C}}-82.8\right)$, Position $3\left(\delta_{\mathrm{H}}-4.01,1 \mathrm{H}\right.$, $b r s)\left(\delta_{\mathrm{C}} 68.8\right)$, Position $4\left(\left(\delta_{\mathrm{H}}-2.88,1 \mathrm{H} d d,(4.1,12.15 \mathrm{~Hz}, 2.84\right.\right.$, $1 \mathrm{H}_{\mathrm{b}} d d,(4.5,12.09 \mathrm{~Hz})\left(\delta_{\mathrm{C}}-28.5\right)$, Position $5\left(\delta_{\mathrm{C}}-157.5\right)$, Position $6\left(\delta_{\mathrm{H}}-5.89,1 \mathrm{H}, d,(2.2 \mathrm{~Hz})\left(\delta_{\mathrm{C}}-95.6\right)\right.$, Position $7\left(\delta_{\mathrm{C}}-157.7\right)$, Position $8\left(\delta_{\mathrm{H}^{-}}-9.97,1 \mathrm{H}, \mathrm{d},(2.2 \mathrm{~Hz})\right)\left(\mathrm{C}_{\mathrm{C}}-96.5\right)$, Position $9\left(\delta_{\mathrm{H}}\right)\left(\delta_{\mathrm{C}^{-}}\right.$ -156.8), Position $10\left(_{\mathrm{H}^{-}}\right)\left(\delta_{\mathrm{C}^{-}} 101.0\right)$, Position 1' $\left(\delta_{\mathrm{H}^{-}}\right)\left(\delta_{\mathrm{C}^{-}}-132.2\right)$, Position 2' $\left(\delta_{\mathrm{H}}-7.09,1 \mathrm{H}, d,(2.2 \mathrm{~Hz})\left(\delta_{\mathrm{C}^{-}}-115.4\right)\right.$, Position $3^{\prime}\left(\delta_{\mathrm{H}^{-}}\right)$

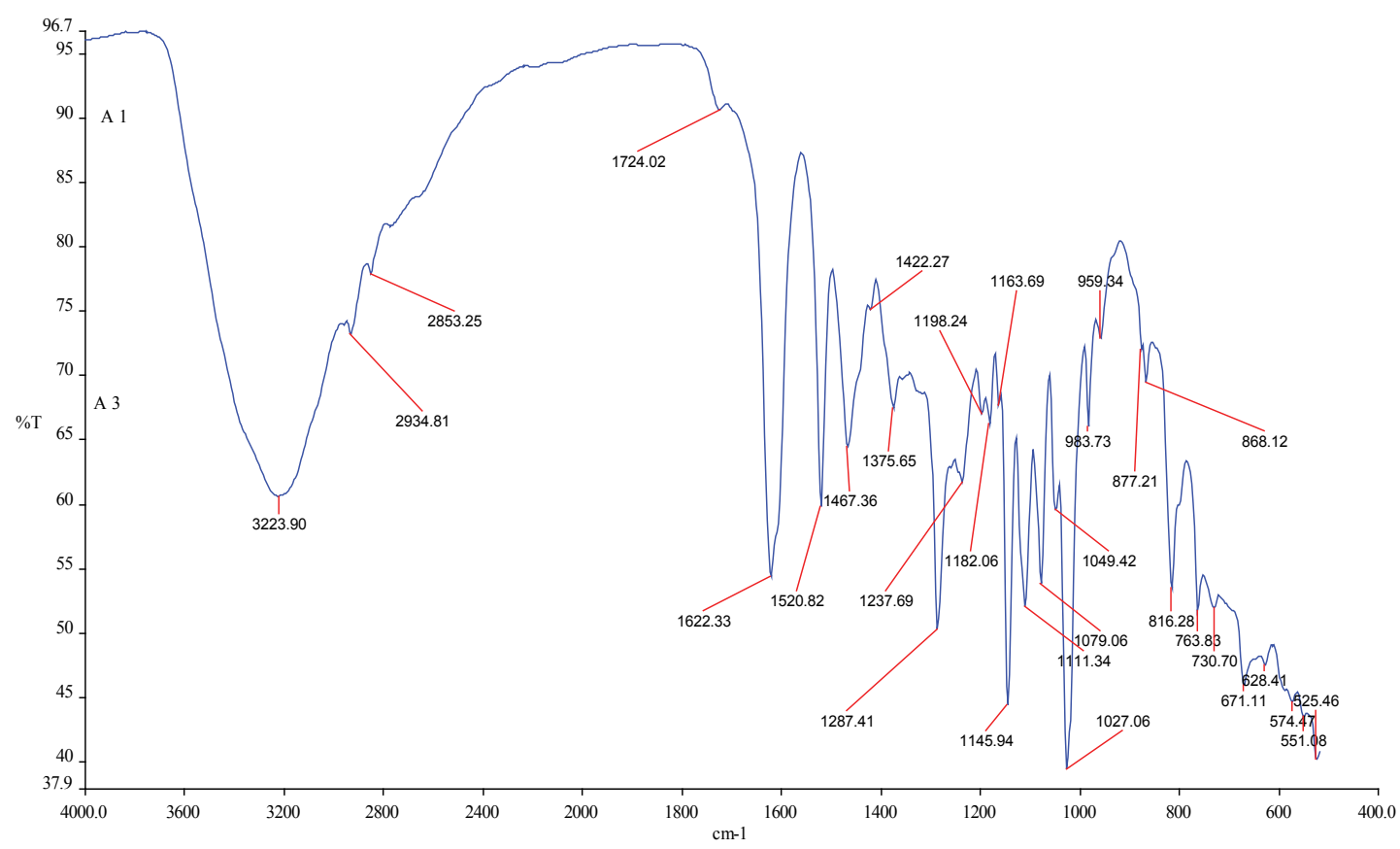

Figure 11: FTIR of Epicatechin. 
Citation: Osuntokun OT, Idowu TO, Cristina GM (2018) Bio-guided Isolation, Purification and Chemical Characterization of Epigallocatechin; Epicatechin, Stigmasterol, Phytosterol from of Ethyl Acetate Stem Bark Fraction of Spondias mombin (Linn.). Biochem Pharmacol (Los Angel) 7: 240. doi: 10.4172/2167-0501.1000240

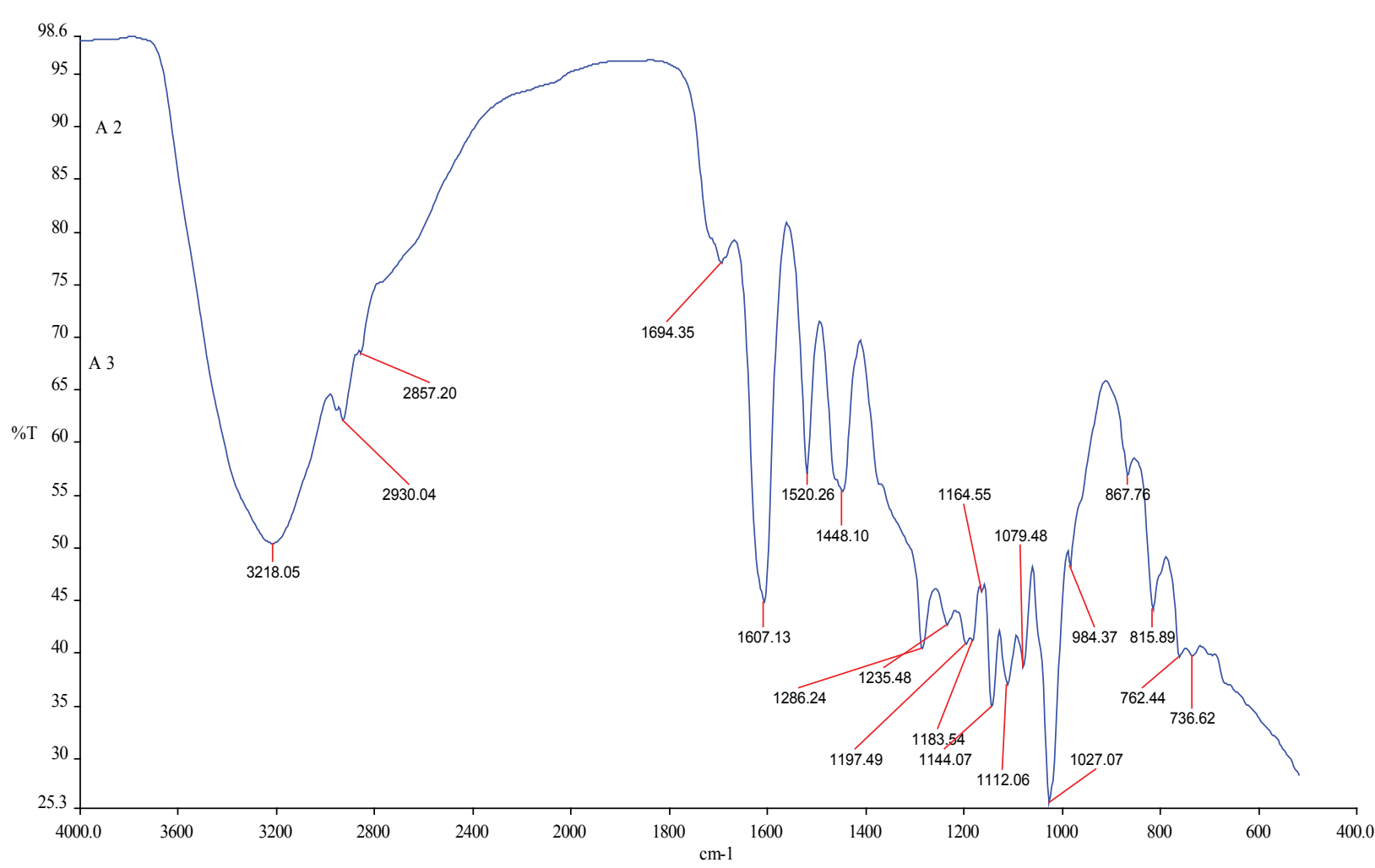

Figure 12: FTIR of Epigallocatechin.

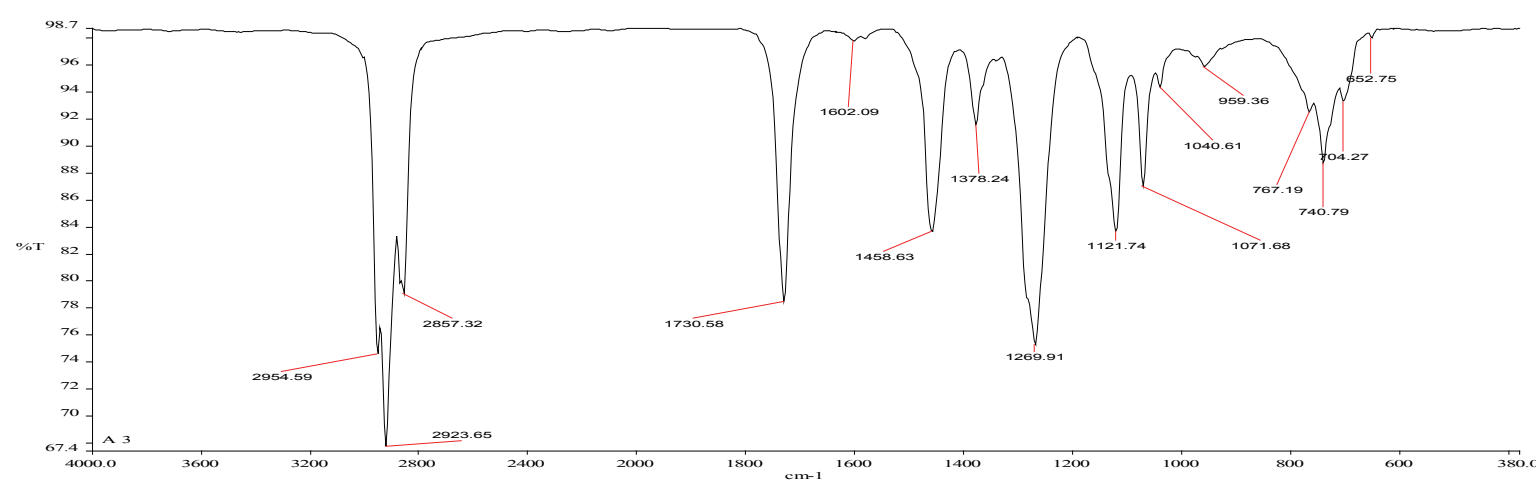

Figure 13: FTIR of Stigmasterol Phytosterol.

$\left(\delta_{\mathrm{C}^{-}}-146.2\right)$, Position 4' $\left(\delta_{\mathrm{H}^{-}}\right)\left(\delta_{\mathrm{C}^{-}}-146.4\right)$, Position 5' $\left(\delta_{\mathrm{H}^{-}}-6.74,1 \mathrm{H}\right.$, $d,(6.0 \mathrm{~Hz})\left(\delta_{\mathrm{C}}-116.3\right)$, Position $6^{\prime}\left(\delta_{\mathrm{H}}-6.79,1 \mathrm{H}, d d,(6.2,1.9 \mathrm{~Hz})\right)$ $\left(\delta_{C}-120.2\right)$, Assignments were confirmed by HMBC, HMQC, DEPT and COSY experiments

\section{Identification of Compound 2 as Epigallocatechin}

This compound was obtained as orange powder and gave a molecular ion peak at $\mathrm{m} / \mathrm{z}$ at 306.07 in EIMS which is compatible with the molecular formula $\mathrm{C}_{15} \mathrm{H}_{14} \mathrm{O}_{7}$. Another fragment ion was found at $\mathrm{m} / \mathrm{z} 139.04$ which resulted from a retro-Diels-Alder (RDA) fragmentation.

The ${ }^{1} \mathrm{H}$-NMR spectrum of $\mathbf{2}$ showed signals due to the presence of a pyrogallol ring at $6.53,2 \mathrm{H}, \mathrm{s}$ ) which was assigned to protons $\mathrm{H}-2^{\prime} / \mathrm{H}-6^{\prime}$ placed on carbons C-2' / C-6' $\left(\delta_{\mathrm{C}} 105.59\right)$ in ring $\mathrm{B}$, a phloroglucinol ring comprising of two meta - coupled protons observed at $\delta_{\mathrm{H}} 5.96(1 \mathrm{H}, \mathrm{d}, J=$ $2.4 \mathrm{~Hz})$ and $5.93(1 \mathrm{H}, \mathrm{d}, J=2.4 \mathrm{~Hz})$ which were assigned to protons $\mathrm{H}-6$ and $\mathrm{H}-8$ placed on carbons $\mathrm{C}-6\left(\delta_{\mathrm{C}} 94.98\right)$ and $\mathrm{C}-8\left({ }_{\mathrm{C}} 94.50\right)$ respectively, two non -aromatic oxygen bearing methane 4.76 , and 4.18 which were

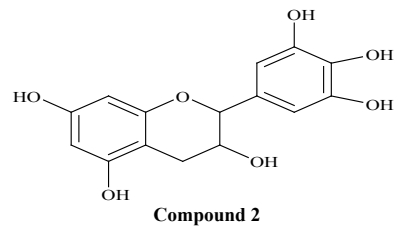


Citation: Osuntokun OT, Idowu TO, Cristina GM (2018) Bio-guided Isolation, Purification and Chemical Characterization of Epigallocatechin; Epicatechin, Stigmasterol, Phytosterol from of Ethyl Acetate Stem Bark Fraction of Spondias mombin (Linn.). Biochem Pharmacol (Los Angel) 7: 240. doi: 10.4172/2167-0501.1000240

Page 8 of 9

assigned to protons $\mathrm{H}-2$ and $\mathrm{H}-3$, a methylene signal at 2.86 and 2.74 which was assigned to $\mathrm{H}-4$. By the combination of ${ }^{1} \mathrm{H}-\mathrm{NMR},{ }^{13} \mathrm{C}-\mathrm{NMR}$, DEPT, HMQC and HMBC spectral analysis, all carbon and proton signals could be definitely assigned.

Based on the above evidence, compound 2 were concluded to be epigallocatechin which was in agreement with the reported literature values [6]. It is a broadly distributed chemical constituent in the plant kingdom and is reported to possess a powerful antioxidant activity [7].

${ }^{1} \mathrm{H}$ - and ${ }^{13} \mathrm{C}-\mathrm{NMR}$ Spectral Data for compound 2 in $\mathrm{CD}_{3} \mathrm{OD}$ at 300 $\mathrm{MHz}$ were listed below.

Ring C- C-2 $\left(\delta_{\mathrm{H}}-4.89,1 \mathrm{H}\right.$, br s $)\left(\delta_{\mathrm{C}} 82.8\right),{ }^{13} \mathrm{C}$ NMR(in acetone- $\left.d_{6}\right)$ 79.4, C-3 $\left.\left.\left(\delta_{\mathrm{H}}-4.01,1 \mathrm{H}, b r s\right)\right) \delta_{\mathrm{C}} 68.8\right),{ }^{13} \mathrm{C}$ NMR(in acetone- $\left.d_{6}\right)-67.0, \mathrm{C}-4$ $\left(\delta_{\mathrm{H}}-2.88,1 \mathrm{H}_{\mathrm{a}} d d,\left(4.1,12.15 \mathrm{~Hz}, 2.84,1 \mathrm{H}_{\mathrm{b}} d d,(4.5,12.09 \mathrm{~Hz})\left(\delta_{\mathrm{C}} 28.5\right)\right.\right.$, ${ }^{13} \mathrm{C}$ NMR(in acetone- $\left.d_{6}\right)-28.8$, Ring A- C-5 $\left(\delta_{\mathrm{H}^{-}}\right)\left(\delta_{\mathrm{C}} 157.5\right),{ }^{13} \mathrm{C} \mathrm{NMR}($ in acetone- $\left.d_{6}\right)-157.1, \mathrm{C}-6\left(\delta_{\mathrm{H}}-5.89,1 \mathrm{H}, d,(2.2 \mathrm{~Hz})\right) \delta_{\mathrm{C}}-95.6,{ }^{13} \mathrm{C} \mathrm{NMR}(\mathrm{in}$ acetone- $\left.d_{6}\right)-96.3, \mathrm{C}-7\left(\delta_{\mathrm{H}}-\right)\left(\delta_{\mathrm{C}-} 157.7\right),{ }^{13} \mathrm{C} \mathrm{NMR}\left(\right.$ in acetone- $\left.d_{6}\right)-157.5$, $\mathrm{C}-8\left(\delta_{\mathrm{H}}-5.97,1 \mathrm{H}, d,(2.2 \mathrm{~Hz})\right)\left(\delta_{\mathrm{C}}-96.5\right),{ }^{13} \mathrm{C} \mathrm{NMR}\left(\right.$ in acetone- $\left.d_{6}\right)-95.7$, C-9 $\left(\delta_{\mathrm{H}^{-}}\right)\left(\delta_{\mathrm{C}^{-}} 156.8\right),{ }^{13} \mathrm{C}$ NMR(in acetone- $\left.d_{6}\right)-157.5, \mathrm{C}-10\left(\delta_{\mathrm{H}^{-}}\right)\left(\delta_{\mathrm{C}^{-}}\right.$ 101.0), ${ }^{13} \mathrm{C}$ NMR(in acetone- $\left.d_{6}\right)-99.9$, Ring B- C- $1^{\prime}\left(\delta_{\mathrm{H}^{-}}\right)\left(\delta_{\mathrm{C}} 132.2\right),{ }^{13} \mathrm{C}$ NMR(in acetone- $\left.d_{6}\right)-131.5, \mathrm{C}-2^{\prime}\left(\delta_{\mathrm{H}}-6.9,1 \mathrm{H}, \mathrm{s}\right)\left(\delta_{\mathrm{C}}-110.5\right),{ }^{13} \mathrm{C} \mathrm{NMR}($ in acetone- $\left.d_{6}\right)-107.0, \mathrm{C}-3^{\prime}\left(\delta_{\mathrm{H}^{-}}\right)\left(\delta_{\mathrm{C}}-146.2\right),{ }^{13} \mathrm{C} \mathrm{NMR}\left(\right.$ in acetone- $\left.d_{6}\right)-146.1$, C-4 $4^{\prime}\left(\delta_{\mathrm{H}^{-}}\right)\left(\delta_{\mathrm{C}^{-}} 132.60\right),{ }^{13} \mathrm{C}$ NMR(in acetone- $\left.d_{6}\right)-132.9, \mathrm{C}-5^{\prime}\left(\delta_{\mathrm{H}^{-}}\right)\left(\delta_{\mathrm{C}^{-}}\right.$ 146.4), ${ }^{13} \mathrm{C}$ NMR(in acetone- $\left.d_{6}\right)-146.1, \mathrm{C}-6^{\prime}\left({ }_{\mathrm{H}}-6.9,1 \mathrm{H}, \mathrm{s}\right)\left(\delta_{\mathrm{C}}-110.5\right),{ }^{13} \mathrm{C}$ NMR(in acetone- $\left.d_{6}\right)-107.0$, (Scheme 2).

Identification of compound 3 as Stigmasterol phytosterol. Compound 3 was obtained as a white crystal. The positive- ion ESI-MS exhibited $[\mathrm{M}+\mathrm{H}]^{+}$at $\mathrm{m} / \mathrm{z} 413$ compatible with the molecular formula $\mathrm{C}_{29} \mathrm{H}_{48} \mathrm{O}$. Its ${ }^{1} \mathrm{H}-\mathrm{nmr}$ and ${ }^{13} \mathrm{C}-\mathrm{nmr}$ spectrum showed three olefinic protons as one triplet at $5.23(\mathrm{C}-6,117.74)$, and two doublets of doublets at $5.16(\mathrm{C}-22,132.46)$ and $5.10(\mathrm{C}-23,129.94)$, a proton germinal with hydroxyl group as a multiplet at $3.60(\mathrm{C}-3,71.45)$. The ${ }^{13} \mathrm{Cnmr}$ spectra showed 29 carbon signals composed of 6 methyl, 6 methylene, 3 olefinic methines, 8 non-olefinic methines and 3 quaternary carbons. The identity of compound $\mathbf{3}$ as stigmasterol was confirmed by comparison of its physical and spectra data with those reported in the literature [8-10]. Stigmasterol is a ubiquitous phytosterol, occurring naturally in a wide variety of plants. However, it is here reported isolated from the plant for the first time.

${ }^{1} \mathrm{H}$ - and ${ }^{13} \mathrm{C}-\mathrm{NMR}$ Spectral Data for compound 3 in $\mathrm{CD}_{3} \mathrm{Cl}$ at 300 $\mathrm{MHz}$ were listed below.

Position $1-{ }^{13} \mathrm{C}(30.18)\left({ }^{13} \mathrm{C}\right.$ NMR-in $\left.\mathrm{CD}_{3} \mathrm{Cl}-31.90\right)$, Position $2-{ }^{13} \mathrm{C}$ (69.26) $\left({ }^{13} \mathrm{C}\right.$ NMR-in $\left.\mathrm{CD}_{3} \mathrm{Cl}-71.45\right)$, Position $4{ }^{-13} \mathrm{C}$ (38.40) $\left({ }^{13} \mathrm{C}\right.$ NMRin $\left.\mathrm{CD}_{3} \mathrm{Cl}-38.42\right)$, Position $5-{ }^{13} \mathrm{C}(146.29)\left({ }^{13} \mathrm{C}\right.$ NMR-in $\left.\mathrm{CD}_{3} \mathrm{Cl}-139.96\right)$,

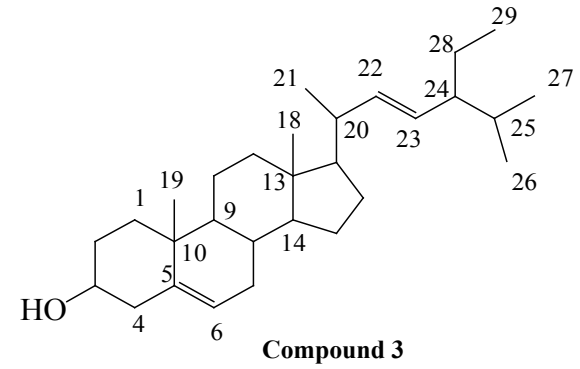<smiles>Oc1cc(O)c2c(c1)O[C@H](c1ccc(O)c(O)c1)[C@H](O)[C@H]2I</smiles>

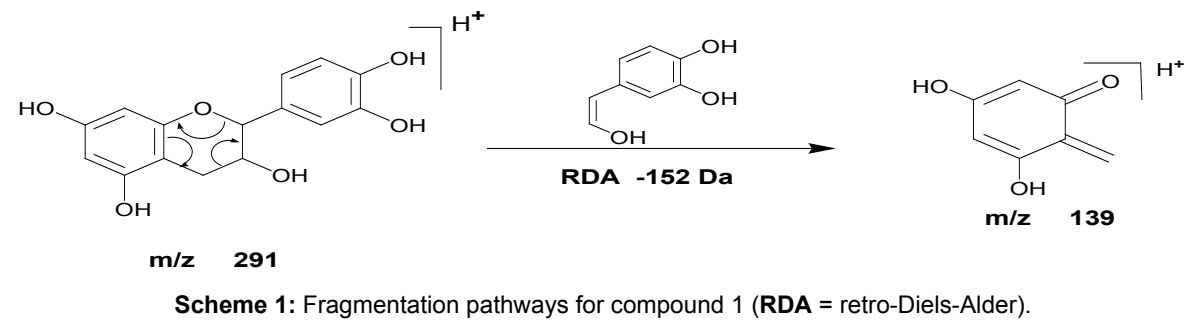

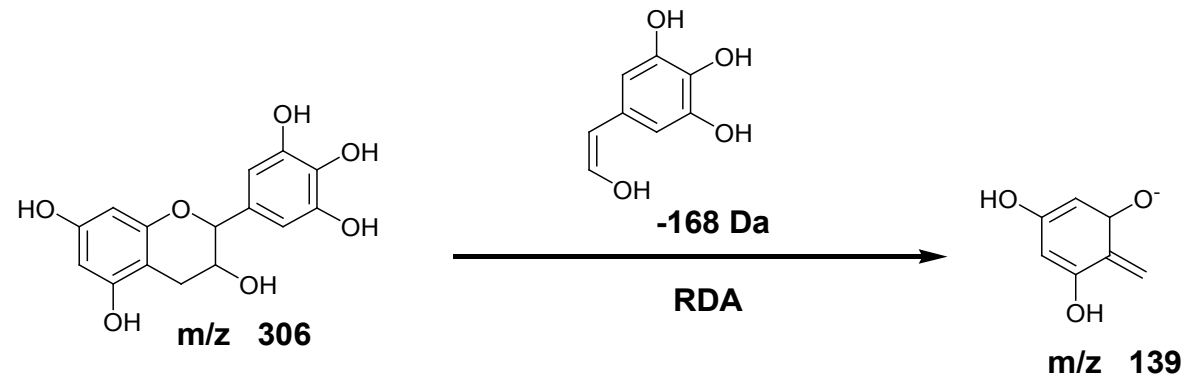

Scheme 2: ESI-MS fragmentation pattern for compound XII (RDA = retro-Diels-Alder fission (-)-epigallocatechin). 
Citation: Osuntokun OT, Idowu TO, Cristina GM (2018) Bio-guided Isolation, Purification and Chemical Characterization of Epigallocatechin; Epicatechin, Stigmasterol, Phytosterol from of Ethyl Acetate Stem Bark Fraction of Spondias mombin (Linn.). Biochem Pharmacol (Los Angel) 7: 240. doi: 10.4172/2167-0501.1000240

Page 9 of 9

), Position $7-{ }^{13} \mathrm{C}(37.66)\left({ }^{13} \mathrm{C}\right.$ NMR-in $\left.\mathrm{CD}_{3} \mathrm{Cl}-37.56\right)$, Position $8{ }^{-13} \mathrm{C}$ (41.12) ( ${ }^{13} \mathrm{C}$ NMR-in $\left.\mathrm{CD}_{3} \mathrm{Cl}-41.20\right)$, Position $9-{ }^{13} \mathrm{C}(48.78)\left({ }^{13} \mathrm{C}\right.$ NMRin $\left.\mathrm{CD}_{3} \mathrm{Cl}-49.88\right)$, Position $10-{ }^{13} \mathrm{C}(31.66)\left({ }^{13} \mathrm{C}\right.$ NMR-in $\left.\mathrm{CD}_{3} \mathrm{Cl}-34.63\right)$, Position $11-{ }^{13} \mathrm{C}(21.65)\left({ }^{13} \mathrm{C}\right.$ NMR-in $\left.\mathrm{CD}_{3} \mathrm{Cl}-21.96\right)$, Position $12-{ }^{13} \mathrm{C}$ (39.34) $\left({ }^{13} \mathrm{C}\right.$ NMR-in $\left.\mathrm{CD}_{3} \mathrm{Cl}-39.88\right)$, Position $13-{ }^{13} \mathrm{C}(42.63)\left({ }^{13} \mathrm{C}\right.$ NMRin $\left.\mathrm{CD}_{3} \mathrm{Cl}-43.70\right)$, Position $14{ }^{-13} \mathrm{C}(56.51)\left({ }^{13} \mathrm{C}\right.$ NMR-in $\left.\mathrm{CD}_{3} \mathrm{Cl}-56.33\right)$, Position $15-{ }^{13} \mathrm{C}(24.08)\left({ }^{13} \mathrm{C}\right.$ NMR-in $\left.\mathrm{CD}_{3} \mathrm{Cl}-23.41\right)$, Position $16^{-{ }^{13} \mathrm{C}}$ (25.00) $\left({ }^{13} \mathrm{C}\right.$ NMR-in $\left.\mathrm{CD}_{3} \mathrm{Cl}-28.88\right)$, Position $17-{ }^{13} \mathrm{C}(54.52)\left({ }^{13} \mathrm{C} \mathrm{NMR}-\right.$ in $\left.\mathrm{CD}_{3} \mathrm{Cl}-55.54\right)$, Position $18{ }^{13} \mathrm{C}$ (12.87) $\left({ }^{13} \mathrm{C}\right.$ NMR-in $\left.\mathrm{CD}_{3} \mathrm{Cl}-12.62\right)$, Position $19-{ }^{13} \mathrm{C}$ (14.56) $\left({ }^{13} \mathrm{C}\right.$ NMR-in $\left.\mathrm{CD}_{3} \mathrm{Cl}-13.42\right)$, Position $20-{ }^{13} \mathrm{C}$ (40.18) ( ${ }^{13} \mathrm{C}$ NMR-in $\left.\mathrm{CD}_{3} \mathrm{Cl}-40.68\right)$, Position $21-{ }^{13} \mathrm{C}$ (21.49) $\left({ }^{13} \mathrm{C}\right.$ NMRin $\left.\mathrm{CD}_{3} \mathrm{Cl}-21.46\right)$, Position $22-{ }^{13} \mathrm{C}$ (132.46) $\left({ }^{13} \mathrm{C} \mathrm{NMR}-\right.$ in $\left.\mathrm{CD}_{3} \mathrm{Cl}-138.55\right)$, Position $23-{ }^{13} \mathrm{C}(129.94)\left({ }^{13} \mathrm{C}\right.$ NMR-in $\left.\mathrm{CD}_{3} \mathrm{Cl}-129.88\right)$, Position $24-{ }^{13} \mathrm{C}$ (51.35) $\left({ }^{13} \mathrm{C}\right.$ NMR-in $\left.\mathrm{CD}_{3} \mathrm{Cl}-51.65\right)$, Position $25-{ }^{13} \mathrm{C}$ (30.99) $\left({ }^{13} \mathrm{C}\right.$ NMRin $\left.\mathrm{CD}_{3} \mathrm{Cl}-32.27\right)$, Position $26-13 \mathrm{C}(21.29)\left({ }^{13} \mathrm{C}\right.$ NMR-in $\left.\mathrm{CD}_{3} \mathrm{Cl}-21.76\right)$, Position 27-13C (19.14) ( ${ }^{13} \mathrm{C}$ NMR-in $\left.\mathrm{CD}_{3} \mathrm{Cl}-19.37\right)$, Position 28${ }^{13} \mathrm{C}(25.23)\left({ }^{13} \mathrm{C}\right.$ NMR-in $\left.\mathrm{CD}_{3} \mathrm{Cl}-25.78\right)$, Position $29-{ }^{13} \mathrm{C}(11.57)\left({ }^{13} \mathrm{C}\right.$ $\mathrm{NMR}$-in $\mathrm{CD}_{3} \mathrm{Cl}-12.44$ ), (Idowu, 2016). All the three pure compounds are well known but reported for the first time in Spondias mombin.

Some authors classified epicatechin as a type of flavonoid which is found in Spondias mombin extract, polyphenols comprises of $30-40 \%$ of extract solids in the herbaceous tree, there are different classes of epicatechin, epigallocatechin [11]. Epicatechin have been proven to have diverse benefits to human health. It reduces the risks of diabetes mellitus and cardiovascular diseases this shows the basic important aspect of Spondias mombin tree, which have pharmalogical effects such as anti hyporlipidemic, anti-inflammatory, antioxidative effect, anticarcinogenic and cytoprotective [7].

EGC arts as a strong inhibitor of HIV replication in cultured peripheral blood cells and inhibition of HIV-1 reverse transcriptase in vitro. EGC binds directly to CD4 molecules with consequent inhibition of $\mathrm{Gp} 120$ binding and inactivate viruses in vitro by deformation of phospholipids [12-14]. It should be mentioned that EC are antioxidant, they are effective scavengers and free radical such a reactive oxygen species (ROS), Reactive nitrogen species and superoxide [15].

Epicatechin and other polyphenols decreases the susceptibility of low density lipoprotein to oxidation which prevents the initiation of artherosclerosis, HIV protein (Tat) and gp120 is known to cause neurotoxicity in human via mechanisms that activate macrophages and glial cells and finally, oxidative stress it can be suggested that epicatechin are neuroprotective by blocking the neurotoxic effects of the HIV protein which cause oxidative stress [16].

However, it should be mentioned that Catechins have an antiprohferative effect on turmor cells as well as inhibiting metastasis and it ability to modulate antioxidant enzymes in vivo. It has been shown to increase the activity of super oxide dismutase and catalase. It also suppresses lipid peroxidation of turmor cells [17].

It must be clearly stated that stigmasterol phytosterol have been shown to lower/reduce blood cholesterol and this lowering may reduce the risk of coronary heart disease. Phytosterols are under preliminary research for their potential to inhibit lung, stomach, ovarian and breast cancers as well as colon and prostate cancers [15].

Apart from lipid peroxidation, epicatechin binds secondary bile acid (Tauro-deoxycholic acid), thus secondary bile has been associated with increased risk of developing colorectal cancer thence these epicatechin from Spondias mombin polyphenolic compound may reduce the risk factor for the developing colorectal cancer [18].

\section{Conclusion}

In conclusion, epicatechin, epigallocatechin and stigmasterol phytosterol isolated from stem bark of Spondias mombim extract has proven itself to be nature's extraordinary therapeutic agent and it has also been proved during the course of this project work. It has also been proved that, Spordias mombin is a potent medicinal plant that its uses and application should be encouraged. More time and attention should be spent on developing it as a sustainable drug for prophylaxis and treatment of complications and diseases.

\section{Acknowledgements}

The authors wish to express their appreciation to all the technical staffs of the laboratory unit of Both the Department of Microbiology, Faculty of Science Adekunle Ajasin University, Akungba Akoko, Ondo State, Nigeria and Obafemi Awolowo University, lle Ife, Osun State, Nigeria for their support and all the technical assistance rendered during the course of this research work.

\section{References}

1. Ajayi IA, Ajibade O, Oderinde RA (2011) Preliminary phytochemical analysis of some plant seed. Res J Chem Sci 1: 58-62.

2. Ayoka A, Akomolafe R, Akinsomisoye O, Ukponmwan O (2008) Medicinal and Economic Value of Spondias mombin. Afr J Biomed Res 11: 29-136.

3. Rodrigues KF, Hasse M (2000) Antimacrobial activities of secondary metabolites produced by endophytic fungi from Spondias mombin. J Basic Microbiol 40: 261-267.

4. Nworu CS, Akah PA, Okoye FB, Toukam DK, Udeh J, et al. (2011) The leaf extract of Spondias mombin L. displays an anti-inflammatory effect and suppresses inducible formation of tumor necrosis factor- $\alpha$ and nitric oxide (NO) $\mathrm{J}$ Immunotoxicol 8: 10-16.

5. Davis AL, Cai Y, Davies AP, Lewis JR (1996) $1 \mathrm{H}$ and 13C Assignments of some Green Tea Polyphenols. Magn Reson Chem 34: 887-890.

6. Agarwal A, Shen H, Agarwal S, Rao AV (2001) Lycopene Content of Tomato Products:Its Stability, Bioavailability and in vivo Antioxidant Properties. J Med Food 4: 9-15.

7. Lin YT, Wu YH, Tseng CK, Lin CK, Chen WC, et al. (2013) Green tea phenolic epicatechins inhibit Hepatitis $C$ virus replication via cyclooxygenase-2 and attenuate virus-induced inflammation. PLoS ONE 8: e54466.

8. Raphael-Leff J (1991) Psychological processes of childbearing. London Chapman \& Hall, fourth edition. Anna Freud centre.

9. Ismail M, Choudhary MI (2008) Compounds isolated from Tannacetum polyce phalum. Turk J Chem 32: 201-204.

10. Idowu TO, Ogundaini AO, Adesanya SA, Onawunmi GO, Osungunna MO, et al. (2016) Isolation And Characterization Of Chemical Constituents From Chrysophyllum Albidum G. Don-Holl. Stem-Bark Extracts and Their Antioxidant And Antibacterial Properties. Afr J Tradit Complement Altern Med 13: 182-189.

11. Wagner H, Ulrich-Merzenich G (2009) Synergy research: approaching a new generation of phytopharmaceuticals. Phytomedicine 16: 97-110.

12. Fassina G, Buffa A, Banelli R, Varnier OE, Noonan DM, et al. (2002) Polyphenolic anti oxidant (-)- epigallocatechin-3-gallate from green tea as a candidate anti HIV agent. AIDS 16: 939-941.

13. Kawai Y, Daniel RA, Errington J (2009) Regulation of cell wall morphogenesis in Bacillus subtilis by recruitment of PBP1 to the MreB helix. Mol Microbiol 71 : 1131- 1144

14. Yamaguchi $K$, Honda $M$, Ikigai $H$, Hara $Y$, Shimamura $T$ (2002) Inhibitory effects of (-)- epigallo catechingallate on the life cycle of human immunodeficiency virus type 1 (HIV-1). Antivir Res 53: 19-34.

15. Osuntokun OT, Oluduro AO, Idowu TO, Omotuyi AO (2017) Assessment of Nephrotoxicity, Anti-Inflammatory and Antioxidant Properties of Epigallocatechin, Epicatechin and Stigmasterol Phytosterol (Synergy) Derived from Ethyl Acetate Stem Bark Extract of Spondias Mombin on Wistar Rats Using Molecular Method of Analysis. J Mol Microbiol 1: 5

16. Nath K, Saini S, Sharma YK (2005) Chromium in tannery industry effluent and its effect on plant metabolism and growth. J Environ Biol 26: 197-204.

17. Tsuchiya H, Tanaka T, Nagayama M (2008) Antiproliferative effects associated with membra ne lipid interaction of green tea catechins. J Health Sci 54: 576-580.

18. Peterlik M (2008) Role of bile acid secretion in human colorectal cancer. Wien Med Wochens Chr 158: 539-541. 